\title{
Key challenges in agent-based modelling for geo-spatial simulation
}

\author{
Andrew Crooks *, Christian Castle, Michael Batty \\ Centre for Advanced Spatial Analysis (CASA), University College London, 1-19 Torrington Place, London WC1E 6BT, United Kingdom
}

\section{A R T I C L E I N F O}

\section{Keywords:}

Agent-based modelling (ABM)

Theory

Validation

Pedestrian models

Residential segregation

Land use models

\begin{abstract}
A B S T R A C T
Agent-based modelling ( $A B M)$ is becoming the dominant paradigm in social simulation due primarily to a worldview that suggests that complex systems emerge from the bottom-up, are highly decentralised, and are composed of a multitude of heterogeneous objects called agents. These agents act with some purpose and their interaction, usually through time and space, generates emergent order, often at higher levels than those at which such agents operate. ABM however raises as many challenges as it seeks to resolve. It is the purpose of this paper to catalogue these challenges and to illustrate them using three somewhat different agent-based models applied to city systems. The seven challenges we pose involve: the purpose for which the model is built, the extent to which the model is rooted in independent theory, the extent to which the model can be replicated, the ways the model might be verified, calibrated and validated, the way model dynamics are represented in terms of agent interactions, the extent to which the model is operational, and the way the model can be communicated and shared with others. Once catalogued, we then illustrate these challenges with a pedestrian model for emergency evacuation in central London, a hypothetical model of residential segregation model tuned to London data, and an agent-based residential location model, for Greater London. The ambiguities posed by this new style of modelling are drawn out as conclusions, and the relative arbitrariness of such modelling highlighted.
\end{abstract}

(c) 2008 Elsevier Ltd. All rights reserved.

\section{Introduction}

Cities are constantly changing and evolving through time and across geographical scales where activities and features change from split second decisions involving local movements such as people walking, the development of land over months and years, the migration of peoples over decades, to the rise and fall of cultures and civilizations over eons. These sorts of problems which involve location and mobility have recently been articulated in much more disaggregate terms than hitherto with their system components or 'objects' being conceived of as agents where their movement takes place in an environment composed of points, areas and networks. Past generations of models of cities have focused on representing such systems as aggregations of populations, rational aggregate behaviour and so on. However the heterogeneous nature of cities makes it difficult to generalise localised problems from that of city-wide problems. To understand urban problems such as sprawl, congestion and segregation, researchers have recently focused on a bottom-up approach to urban systems, specifically researching the reasoning on which individual decisions are made.

\footnotetext{
* Corresponding author. Tel.: +44 (0) 207679 1782; fax: +44 (0) 2078132843. E-mail addresses: andrew.crooks@ucl.ac.uk (A. Crooks), c.castle@ucl.ac.uk (C. Castle), m.batty@ucl.ac.uk (M. Batty).
}

One such approach is agent-based modelling (ABM) which allows one to simulate the individual actions of diverse agents, measuring the resulting system behaviour and outcomes over time. The big difference between these new approaches and the more aggregate, static conceptions and representations that they seek to complement, if not replace, is that they facilitate the exploration of system processes at the level of their constituent elements. The development of ABM is not without its problems and this paper will seek to identify these, posing them as key challenges to be addressed in fashioning these models to make them scientifically relevant and policy applicable (Axelrod, 2007).

We begin by posing seven key challenges which range across the spectrum of theory to practice, hypothesis to application and then illustrating how these challenges can be addressed with three agent-based models, loosely coupled to geographic information systems (GIS) which we are currently working on. Castle (2007a) has been working with a pedestrian model of evacuation dynamics at Kings-Cross/St. Pancras in central London. Crooks (2007) has been working with a hypothetical Schelling-type residential segregation model at a coarser spatial scale tuned to London data which deals with processes of residential mobility over longer time periods. Batty has been working with a land use transportation model of Greater London built along traditional spatial interaction principles with many large zones at the census tract scale but generalised to treat each trip maker as an individual agent. The scale is greater than the first two but the dynamics is more like that of 
the pedestrian model dealing with movements that take place over short periods of time. While there are many agent-based models we could have chosen to demonstrate how these challenges are being addressed, these applications were chosen for several reasons. Firstly, they highlight the current state of ABM and GIS integration. Secondly, they illustrate how agent-based models can be developed through the use of toolkits or built up from scratch, and thirdly the ways in which different representations of space (vector, raster, networks) and scales of geography can be used. Thirdly, they show different application domains and the difficulty in addressing all the challenges posed in a single modelling endeavour.

The principles and concepts of ABM are well known and have been used in a diverse range of applications (see, Castle \& Crooks, 2006, for a review). But unlike earlier mathematical models of urban phenomena (Batty, 1976), these models are much more generic, fashioned according to ABM principles. Despite their many advantages, as a tool for simulating micro-diversity, emergent properties, and process dynamics, ABM did not begin to feature prominently in social simulation and geographical information science until Epstein and Axtell (1996) demonstrated that the notion of modelling individuals making up an idealised society in space could be extended to growing entire artificial cities. The use of $\mathrm{ABM}$ for experimenting and exploring geographical phenomena however is still in its infancy (see, Benenson \& Torrens, 2004; Brown, Riolo, Robinson, North, \& Rand, 2005; Parker, 2005 for sample applications) and thus our focus here is on identifying key challenges to the development of such models. This makes their applicability somewhat different to the previous generations of urban model, in that modellers need to explicitly consider these challenges before they embark on such simulations.

\section{Key challenges}

The seven challenges that we see as important to the development of agent-based models involve the following: the purpose for which the model is built, the extent to which the model is rooted in independent theory, the extent to which the model can be replicated, the way the model might be verified, calibrated and validated, the way model dynamics are represented in terms of agent interactions, the extent to which the model is operational, and the way the model can be communicated and shared with others. We do not consider this to be an exhaustive list but it is a beginning (and more general challenges for creating spatially explicit agent-based models are illustrated in Section 3). Such challenges have been identified before (see particularly Axelrod, 2007), but here we will address them in turn, identifying each major issue and demonstrating them, where appropriate, with actual applications. ${ }^{1}$ While these challenges are reflected within all modelling endeavours and experienced model builders consider them as quite basic, this paper seeks to comprehensively synthesise and reflect on these issues, making them specific to agent-based modellers, and thus alerting them to the pitfalls of past generations of model. Our purpose is to pose the critique and to foster discussion on potential ways to meet these challenges.

\subsection{The purpose of the model}

When computer models were first constructed for urban systems, they were built for testing the impacts of urban plans and policies rather than for scientific understanding per se (Batty,

\footnotetext{
${ }^{1}$ Why seven challenges? We have taken our cue from Axelrod (2007) who defined five such challenges. We consider the seven we have defined to be a useful and manageable shorthand for the problems facing the field of ABM.
}

2008). The argument being: given a good theory, a model would be constructed which would then be validated and if acceptable, used in policy making (Batty, 1976). This argument has been relaxed in the last two decades and now models are built to explore all stages of the theory-practice continuum. This has occurred because the certainty of science has come under fire. The idea that the computer represents the scientist's laboratory is an attractive notion but when it comes to control of inputs and parameters, most social systems cannot be represented in a form that guarantees any measure of closure. The difficulties and failures of earlier generations of urban model, for example, demonstrate that however good the fit of the model is to reality and to theory, there always seem to be features that are missing.

ABM relaxes all these assumptions and most social science simulations do not focus on policy applications. It is more generic, more of a style of modelling than a type of model and is largely independent of theory and practice (North \& Macal, 2007). Thus the purpose of any particular model type will depend on issues that are often beyond its generic principles. In fact, whether or not ABM is appropriate for the theory, its applications, the policies involved or the design of systems that the model might be built to inform, cannot be guessed in advance. Thus only when we broach particular problems and develop particular models, do these issues become clear. We cannot anticipate how good a model will be until it is specified and implemented and thus the quality of a model is independent of the style of modelling adopted.

Frequently in ABM, the actual purpose and position in this scientific process is unclear largely due to the changing conceptions of how to do science and the fact that agent-based models deal with systems that are complex, open-ended, hence emergent and thus exhibit novelty and surprise. However, a model is only as useful as the purpose for which it was constructed and for agent-based models, this needs to be clear. A model has to be built at the right level of description for every phenomenon, using the right amount of detail for the model to serve its purpose (Couclelis, 2002). This remains more art than a science (Axelrod, 2007) but this is using the term science in its narrow sense for there is as much art in science as science in art. The purpose of agent-based models range from the explanatory to the predictive (see, Castle \& Crooks, 2006) with prescriptive and design models of increasing importance. There is less focus on policy and prescription with this style of simulation than in previous, more aggregate modelling.

\subsection{Theory and model}

Models should be based on theory and the traditional role of a model in the social sciences is a translation of theory into a form whereby it can be tested and refined. In this sense, a computer model provides a computer laboratory for virtual experimentation, and hence a vehicle for refining theory through 'what if style experiments and sensitivity testing. In fact as scientific method has blurred from this classical tradition, models are increasingly being used to develop theory. In fact, the term theory has fallen out of favour in many contexts as models themselves contain theories. Our concern here however is that the theoretical implications of many agent-based models remain implicit and hidden, often covered by a thick veil of ad hoc assumptions about structure and process as well as a veneer of software interfacing. In many models, it is hard to figure out what they are for as they are simply additional applications of some simple structure which is tweaked for the local context and application. Domain knowledge is often lacking. Increasingly ABM is considered generic, independent of any particular field or application, and hence subject to use for any purpose that arises. In short, the scientific standards of the past are often buried in ad hoc model development. 
We do not believe that 'theory' should necessarily be independent of 'model' for we are well aware that new styles of model now embrace theory in quite a different manner from those hitherto. But we do consider that in developing good models, there needs to be recognition that many styles of theorising and thinking must be brought to bear on model construction. For example, in our understanding of urban spatial structure, there is a long heritage of location theory in the urban and regional economics domain with this theory being reflected in the equilibrium micro-economics of the individual and the firm. This theory has produced many important insights and in any agent model of residential development, say, we might expect such issues to be reflected. In short, what we are saying is that the domain of model and theory is now considerably wider than at any time in the past and $\mathrm{ABM}$ must respond to such complexity.

\subsection{Replication and experiment}

It is a canon of scientific inquiry that a theory that withstands the test of time is more likely to inform our understanding than one that can be easily refuted. This inductive hypothesis suggests that the more confirming instances of a theory, the stronger it becomes. This is the quest for generalisation in that a theory that works for one set of circumstances must work for any other and as long as the theory is not refuted, it remains 'true'. Of course, all this has been turned on its head by the notion that theories cannot be confirmed but only falsified and that even a theory that withstands many confirmatory instances and applications has no greater probability of being true than any other. It only takes one falsification to sink it.

Nevertheless, our intuition suggests no matter how wrong this might be, our confidence in a model (or its theory) always increases the more confirming instances we have of its successful application. To pursue this, we need to replicate the model in independent situations. This is rarely done in the social sciences largely because of the difficulties in controlling all the variables that pertain to a particular situation, thus making it almost impossible to ensure comparability in terms of applications ${ }^{2}$. A more limited quest which we discuss below is to make sure that the model can be verified in different laboratory situations, with different software for example, rather than different data. This is a much lesser quest than full validation against data. A reverse form of replication involves testing different kinds of models or different variants of the same model, for example, in different software systems, on a standard data base. This is a process referred to as 'docking' (Axtell, Axelrod, Epstein, \& Cohen, 1996) as we have greater control over the model than the data; hence keeping the data fixed and varying the model gives some insight into the robustness of each model. In cases where similar models have been compared on standard data sets as in the case of urban land use transportation models it was found that many idiosyncratic decisions made by the modellers with respect to their different models and in terms of the way the data was configured and defined in different places, made comparisons almost impossible (Webster, Bly, \& Paulley, 1988). This rather dismaying conclusion was for parsimonious models of a rather narrow genus where applications were quite standard in terms of the data required, rather than the much more speculative and pedagogic models that are beginning to characterise the field of ABM.

\footnotetext{
${ }^{2}$ However it is worth noting that this challenge is being met by slow, painful but progressive attempts at devising ontologies and protocols for model comparison such as the ODD (overview, design concepts, details) protocol proposed by Grimm et al (2006). A worked example using three land use and cover change agent-based models can be seen in Polhill, Parker, Brown, and Grimm (2008).
}

\subsection{Verification, calibration, and validation}

"Verification is the process of making sure that an implemented model matches its design. Validation is the process of making sure that an implemented model matches the real-world." (North \& Macal, 2007, pp. 30-31). Verification is thus as much a matter of testing the logic of the model through its computer programme as testing its formal logic. It involves checking that the model behaves as expected which is something that is often taken for granted. It is sometimes referred to as testing the 'inner validity' of the model (Brown, 2006) but we will not use this phraseology here as it tends to confuse verification (which does not involve any external data) with validation. Validation relates to the extent to which the model adequately represents the system being modelled (Casti, 1997) and in this sense, it involves the goodness of fit of the model to data. However, the validity of a model should not be thought of as binary event (i.e. a model cannot simply be classified as valid or invalid); a model can have a certain degree of validity which of course is encapsulated by various measures of fit (Law \& Kelton, 1991).

Validity can thus be ascertained by comparing the output of the model with comparable data collected from a real-world system using various statistics over which there is usually quite intense debate. The question of what best statistics to use in model fitting is something that has dominated the literature on models of land use and land cover, for example (Pontius \& Malanson, 2005). There are also qualitative evaluations of model validity that might be made. Mandelbrot (1983) argues that good models which generate spatial or physical predictions that can be mapped or visualised must 'look right'. Axelrod (2007) suggests that to understand the output of an agent-based model, it is often necessary to evaluate the details of a specific simulation 'history' and this too is usually a qualitative matter.

In contrast, calibration involves fine-tuning the model to a particular context and this means establishing a unique set of parameters that dimension the model to its data. This is not validation per se but calibration can often involve validation because the parameters are often chosen so that performance of the model related to data is optimal in some way, in terms of some criterion of goodness of fit, for example. This is a large subject area and suffice it to say, many if not most agent-based models suffer from a lack of uniqueness in parameter estimation due to the fact that their assumptions and processes tend to outweigh the data available for a complete assessment of their goodness of fit.

Concerns have been raised pertaining to verification and validation by numerous researchers. Batty and Torrens (2005) write that with respect to developing traditional models, two rules have been taken as central to the process of developing good models in the social sciences. The first is the rule of parsimony - Occam's razor which suggests that a better model is one which can explain the same phenomena with a lesser number of intellectual constructs. The second principle relates to independence in validation. A theory which is induced using one set of data needs to be validated against another independent set, and this relates to our earlier discussion about replication. While it is sometimes possible to achieve this with traditional models, this is not the case for models developed using ABM principles, particularly where this involves human systems which evolve over time. Modellers are embracing increasingly diverse and richer model structures containing large numbers of parameters. Often with traditional models, parsimony is reflected in the linkage of dependent and independent variables while agent-based models have multiple causes which display heterogeneity of processes that are impossible to observe in their entirety (Batty \& Torrens, 2005). Thus these new model structures are never likely to be validated in any complete sense against data; they are too rich and data needed to test them too poor (Batty, Steadman, \& Xie, 2006). 


\subsection{Agent representation, aggregation and dynamics}

In spatial systems, what constitutes an agent is a critical issue in that the term can be applied to any aggregation of objects at any spatial scale and across different time horizons. Moreover it need not be restricted to human objects but might pertain to any object that exists in space and/or time (Batty, 2005). Some of these issues of representation are clarified in the examples that we introduce below, particularly in the way we represent their applications and outcomes using various forms of graphic. The scale of agents is also an issue as the finer the scale, the less ambiguous the definition, although we appreciate that this is contentious. This means that there are greater difficulties in specifying rules for defining agents which are aggregations of lower level units, i.e. groups within a human population, or defining abstracted agents such as a forest or a farmer or a city which pertain to models that in themselves are generic. In particular as we aggregate, we can unwittingly change the kinds of processes that agents enable, the mobility intrinsic to their location, and the scale at which they exist. It is thus more and more difficult to define relevant processes as these too are aggregations of lower level routines and behaviours. Aggregation can thus confuse our identification of coherent patterns that make sense in terms of basic human decision-making.

Another issue involves the sheer number of agents and the sheer number of attributes and processes that they are engaged with. Like all systems that deal with interactions and networks, the size of the computation usually rises as the square of the number of agents, if not faster, and there are always limits on our ability to deal with such exponentiation. Sampling is often a favourite strategy to deal with multitudes but we must be cautious about proposing models that seek to represent behaviour at its most elemental level and then simplifying this back through taking samples. Sampling is not a well-developed art in ABM as yet. Moreover choices are necessary in terms of the number of agents and processes which are reflected in the software used, the computation time involved, and of course the ability to get data that matches the specification of the model. In general, most agentbased models are tested against a fraction of data that could be applied to them in that many implicit and explicit assumptions about behaviours cannot be observed as data does not exist. This reflects issues about validation and calibration which we have already noted above as our fourth challenge.

\subsection{Operational modelling}

Making agent-based models operational means moving them to the point where they are configured as simulation models and running them so that they might produce outcomes. In the past, most models have been programmed from scratch and although this keeps the application in touch with theory, it makes the ability to generalise the model to other situations, to replicate the model that is, difficult as the study previously referred to by Webster et al. (1988) indicated. What has happened with ABM is that because this implies a generic approach, various software are now evolving that like GIS, are being used to enable such generic applications. As always, the extent to which generic software can be well-tuned to specific situations will vary according to the application and its complexity, but besides the advantages of consistency and modularity that such software enables, it is always limited in its applicability.

In terms of $\mathrm{ABM}$ as in other areas of simulation and representation, such software enables modellers to adapt it to their problem context, implementing their model through high level scripting, for example, which the software usually allows. This opens up models to a wider community of scholars than hitherto but it also forces modellers without the skills or resources to develop their own models from scratch to meet constraints posed by the software. This can be a key problem when limits posed by the software on the numbers and representation of agents occur. Nevertheless, the development of geo-spatial agent-based models can be greatly facilitated through the use of simulation/modelling systems such as Swarm, Repast, NetLogo, etc. (Castle \& Crooks, 2006). They provide reliable templates for the design, implementation and visualisation of agent-based models, allowing modellers to focus on research (i.e. building models), rather than building fundamental tools necessary to run a computer simulation (Railsback, Lytinen, \& Jackson, 2006; Tobias \& Hofmann, 2004).

\subsection{Sharing and dissemination of the model}

The last challenge involves how we might communicate and share agent-based models with all those who we seek to influence and whom we believe that such modelling will inform. In the past before the development of intensive and all pervasive computation, communicating models was mainly through discussion, simplification and visualisation, through pedagogy in all its various forms. Clearly visualisation is one of the keys to such sharing in that with digital models, their structure is easily amenable to visualisation. Of course spatial outcomes can be mapped and this is a key medium for dissemination as well as for validation and other aspects of the simulation process. But model structures can be described visually while the process of running the model, calibrating it, and examining its inputs and outputs can be presented visually even while the model is running.

A good example of the power of such sharing is embodied in the current model-building capability within the GIS software ArcGIS (Maguire, 2005) which offers an interesting way of involving those who are not expert in simulation model construction. In fact much of the software that is now being evolved not only communicates and shares the modelling process and its outcomes with various non-expert participants but also enables non-experts to participate in the actual model construction. The other face of this revolution is the development of procedures for disseminating this kind of visualisation and model-building process to whoever has an internet connection. The development of online laboratories - collaboratories for example - where model building and users engage in mutual and shared development activities although their infancy are very much on the horizon. The MOSES model at Leeds is a good example of the potential of this kind of activity (Birkin, Turner, \& Wu, 2006). The development of web sites where many users develop agent-based models such as NewTies (Gilbert et al., 2006) is another example of how this field is developing into a more sharing mode where collaboratories hold out great promise for new advances in social simulation.

\section{More general challenges}

To conclude our catalogue of challenges, we will briefly focus on more general issues in creating spatially explicit agent-based models before presenting various examples. While GIS is a particularly useful medium for representing model input and output of a geospatial nature, GIS are not well suited to dynamic modelling (Maguire, 2005) such as ABM. In particular, there are problems of representing time (Langran, 1992; Peuquet, 2005) and change within GIS (Longley, Goodchild, Maguire, \& Rind, 2005). To address these problems, numerous authors have explored linking (through coupling or integration/embedding) a GIS with a simulation/modelling system purposely built, and therefore better suited to supporting the requirements of ABM (e.g. Brown et al., 2005; Westervelt, 2002). 
ABM focuses on individual objects or categories, and thus the progress currently being made in the use of disaggregate data is an essential determinant of their applicability (e.g. Benenson, Omer, \& Hatna, 2002). Increased computer power and storage capacity has made individual-level modelling more practical with a good example seen in the evolution of pedestrian modelling (see, Galea \& Gwynne, 2006) where there has been a concerted movement from aggregate to individual-level modelling. However, limitations still remain when modelling large systems. For example, large and refined datasets of high-resolution information now exist for initialising agent-based models for urban simulations. GIS are capable of encoding these datasets into forms that provide the foundations for such simulations along with providing spatial methods for relating these objects based on their proximity, intersection, adjacency or visibility to each other.

One major stumbling block is that there is potentially too much detail in these data for the current generation of computers to deal with when application to entire cities rather than just small areas are made. Thus agent-based models can suffer from similar limitations to those of the first generation of urban models developed in the 1960s (Lee, 1973). However, this might be overcome by considering the level of abstraction needed to examine the phenomena of interest for all the available detail is rarely needed while a series of smaller models could be created by examining specific aspects of the system. Second there is the lack of personal individualised data for the present and the past. Sometimes access to more personal data can be obtained from commercial sources (see, Benenson et al., 2002) or synthetic populations can be generated through micro-simulation techniques (Birkin et al., 2006) but dynamic, individualised data is in general a major problem which will continue to influence the development of such models in the foreseeable future.

\section{Applications}

In the following section, we demonstrate three very different operational agent-based models in terms of dynamics (from seconds to years), scale (from micro to macro) and application domains. Each of these applications is currently under development and to an extent they demonstrate how we are trying to address the challenges outlined above. It is impossible for us to give a detailed, replicable description of each model in the confines of this paper. However we identify where further information about each model can be found and for each application we give a brief introduction and description relating to the challenges where appropriate.

\subsection{A pedestrian model for emergency evacuation}

The first of our models is based on simulating pedestrians exiting the King's Cross/St. Pancras underground station. In 1987, the station was devastated by a major fire with considerable loss of life and the current redevelopment is still taking into account the past safety recommendations. In addition, the station must cope with the projected increase in future passenger demand from the new Eurostar terminal and the projected $1,05,000$ people who will use the station during the morning peak (7-10 am) during the 2012 Olympics. The model that was built is part of the appraisal by Camden Primary Health Care Trust who are responsible for the allocation and positioning of key emergency functions and facilities (e.g. ambulance loading point(s), casualty clearing station(s) to which the injured can be taken, etc.) in the event of future emergency incidents within the underground station complex. The purpose of the model is to predict the likely evacuation dynamic (i.e. total evacuation time (in seconds)), usage of evacuation routes, and the conditions experienced by passengers (e.g. crowd density) given evacuation scenarios, future travel demand, and fluctuations in passenger use at different times of the day and week.

With this well defined policy context, the model (which we refer to as the King's Cross Pedestrian Evacuation Model - KXPEM) is built on a theory of movement associated with evacuation (Castle, 2007b). Models such as this have been built by several researchers and are quite widely applied. They are the closest of all agentbased models to traditional scientific models which are testable against data and are capable of being replicated in different situations (see, Castle(2007a) for further details). This is largely because of the simplified behaviour of the agents (who optimise their utility associated with least cost movement paths), although the context of the problem is such that cultural and institutional differences, differences in geometric construction of facilities, and the standard emergency practice makes each application unique. Sharing the model is essential and although the application is still in desktop form, several groups involved in the Kings Cross evacuation scenarios, are involved in the model design and use. In short, this is typical of the best examples of ABM tuned to a real problem as currently exists.

The model was programmed in Java which is used in relation to the agent-based simulation/modelling toolkit Repast rather than using off-the-shelf packages such as Legion, buildingExodus, etc. (Castle \& Longley, 2008). The conceptual model of KXPEM was based on the theory and principles of pedestrian evacuation modelling identified by Castle (2007b) which focus on issues such as geometric enclosure representation, occupant and enclosure perspective, speed and direction of occupant movement, and the behavioural perspective of pedestrians, all critical factors that influence its design. As pedestrian movement involves representing a relatively well-behaved but heterogeneous population of occupants, the enclosure representation is based on a regular $(50 \mathrm{~cm} \times 50 \mathrm{~cm})$ lattice representation.

With regard to agent representation, each pedestrian is defined as a movable object, each agent is aggregated into a group, groups are definable in terms of age, gender, and passenger type, and all this permits a heterogeneous population of passengers. Furthermore, KXPEM permits either a global or individual perspective of the station layout as pedestrians are defined as either occasional or regular passengers. The calculation of a regular passenger's exit route-choice is based on the assumption of prior knowledge of the station layout, and habitual use. Conversely, occasional passengers are programmed with limited knowledge of the station layout. Their exit route-choice is calculated based on the assumption they will follow emergency signage to exit the station. The speed at which a pedestrian can walk is dependent upon their available space (i.e. density of pedestrians within a local area), conditions of their local environment (e.g. surface terrain) and characteristics of the individual (e.g. age and gender). Four secondary data sources for pedestrian walking speed, have been incorporated into the model to explore their effect on simulation outcomes: these come from Hankin and Wright (1958), Ando, Ota, and Oki (1988), Fruin (1971) and Predtechenskii and Milinskii (1978). Exit route-choice and way-finding are defined by cost surfaces, akin to many other pedestrian evacuation models that adopt a regular lattice approach to enclosure representation. Based on the assessment criteria of pedestrian evacuation models, KXPEM adopts a rule-based approach to simulate occupant behaviour. The rules that determine the response of each pedestrian when confronted with a decision are a combination of deterministic and stochastic responses based on information derived from the literature (Castle, 2007a).

The validation and calibration of KXPEM was an intensive process. Floor plans from computer aided design (CAD) of the station were used to ground the model with respect to the accuracy of enclosure's layout and therefore its capacity. In line with the 
model's purpose, cost surfaces were developed to explore three evacuation scenarios. Two scenarios are defined by the UK Health and Safety Executive (HSE, 1996) to assist in the design and analysis of escape capacity from railway stations. These are, a train on fire at a platform, and second, a fire within a station. The third scenario permits the simulation of pedestrians from the station without incident. In particular, periods of observation were made of pedestrian movement at the station in order to calibrate the cost surfaces used to specify direction of pedestrian movement and route-choice. In addition, surveys at the station were used to determine passenger volume and characteristics (e.g. age, gender and passenger type at different times of the day and week), in order to specify these parameters within the model. In terms of enabling the model to be built and used, an advisory panel of key stakeholders to Kings Cross redevelopment was set up to facilitate its development, in particular to gain access to necessary information, often not in the public domain (survey data and CAD floor plans, for instance), and to advise on the development and calibration of the model. Once developed, predictions were disseminated amongst this group, but more general dissemination to a wider audience is restricted due to security issues.

Typical of most ABM and the other two models presented below, the development of KXPEM involved an iterative process where model verification was based on many iterations of the system. Each version represented a major progression and backup point. A descriptive log of each programming progression was kept in case the author needed to reverse any changes. Unit testing was undertaken after every adjustment to the programming code. As the model became more complex, small and quick changes to the code took several hours to verify. Unit testing was achieved through Eclipse integrated development environment (IDE) debug mode, print lines, and by visual analysis of the model. Following this meticulous regime of verification, confidence was gained in the model, specifically in terms of model processes taking place at the right time, and each process occurring in the manner in which it was intended. KXPEM was designed and developed for predictive purposes but information regarding past evacuation drills at the station and detailed empirical data on passenger flow was largely absent. In this sense, the model was not fully validated in the traditional manner against real-world data, and thus it is better suited for exploratory purposes at present. The visualisations in Fig. 1 not only reveal the process of building and testing the model but the kind of outputs that non-expert users can relate to.

\subsection{A model of residential segregation}

Our second application involves an extension to Schelling's, (1971) classic model in which individuals with very mild preferences to live amongst their own kind generate highly segregated districts when they move in response to any mismatch between their preferences and the configuration of their own and different types in their immediate neighbourhood. The purpose of this model is to explore the impact of space and geometry on such a process and is simply a pedagogic demonstration, of how individuals react to one another with respect to their preferences. As far as the model is grounded in theory, it is the notion that individual action does not lead to any collective welfare, quite the opposite. It shows how unfettered and uncoordinated actions at the individual level lead to unexpected outcomes that are collectively undesirable, mild preferences revealing what are often quite wrongly judged to be extreme preferences. Its theoretical basis is commonsense, and the model is uncluttered with additional variables that might affect segregation - for example, how economic factors may contribute to racial segregation based on systematic income differences across.
The model departs from other models which either explore or extend Schelling's original insights (e.g. Benenson et al., 2002; Bruch \& Mare, 2005; O'Sullivan et al., 2003) which are all based on the regular partitioning of space (e.g. cells or polygons) to represent the location of households. The focus here is on how different conceptions of spatial organisation affect the process of segregation with the model allowing agents to move anywhere within the urban environment (i.e. movement is not restricted to discrete cells or areas as the model uses a continuous representation of space - vector rather than raster space). The model explores how segregation emerges as agents move to new locations, and how segregated areas grow and decline over time. In this sense, it makes Schelling's model much more explicitly geographical than any other applications to date but it is easy to replicate and is an ideal basis for experimentation. Its pedagogic quality and the ease with which it can be shared acts as a demonstration of how complex, unexpected, and surprising patterns emerge from simple foundations. However, as with other segregation models the time frame within the model is hypothetical but could be considered as being configured in yearly intervals.

In GIS terms, the model comprised two vector layers - the urban environment represented as a series of polygons, and four types of agents (red, blue, green and white) represented as points. It is the information held within fields of the environment layer that is used to create the agents. The distribution of four types (representing ethnic groups, say) of agent as observed through aggregate census population counts form the initial starting conditions of the model. Fig. 2A represents four wards in the City of London each with their own attribute information stored in a data table where each row relates to a specific ward (e.g. ward 1 has a population of ten red $^{3}$, five blue, four green and two white agents). The model reads this data and creates an environment polygon for each ward and for the desired agent population based on data held in the fields as in Fig. 2B. Note that the underlying colour of the polygon (ward) always represents the predominant social group in the area. This model is designed to work on many different geographical scales (e.g. boroughs and OS MasterMap TOIDs) without the need for model reconfiguration as we show in Fig. 3. This functionality was created so that the model could be easily replicated in other areas in the quest to allow the modeller to see if the same rules can be applied to different areas and at different scales. Replicability is one of the key challenges we identify above and using modular software such as Repast in which the model is scripted, enables such flexibility in application.

In this model, agents only move if they find themselves in a minority which we have set as being less than a certain percentage of the same kind in their area (neighbourhood). While Schelling's original segregation model is an excellent example explaining residential dynamics, there are limitations. First, reality is much more complex and for this reason, the model has been extended in several ways, particularly in defining more than two groups. For example, within London, there are numerous types of ethnic or socioeconomic group and thus this extension explores the impact of four different types of agents (although the model can permit any number). Each agent has a preference related to residential contact (co-residence) with members of each other group. Not only are we interested in how patterns of segregation evolve over time but how this pattern changes with the introduction of new agents and the death of older agents. Thus the model allows for the addition and removal of agents which has an important effect on the pattern of segregation seen within urban areas.

\footnotetext{
${ }^{3}$ For interpretation of color in Figs. 2 and 5 the reader is referred to the web version of this article.
} 


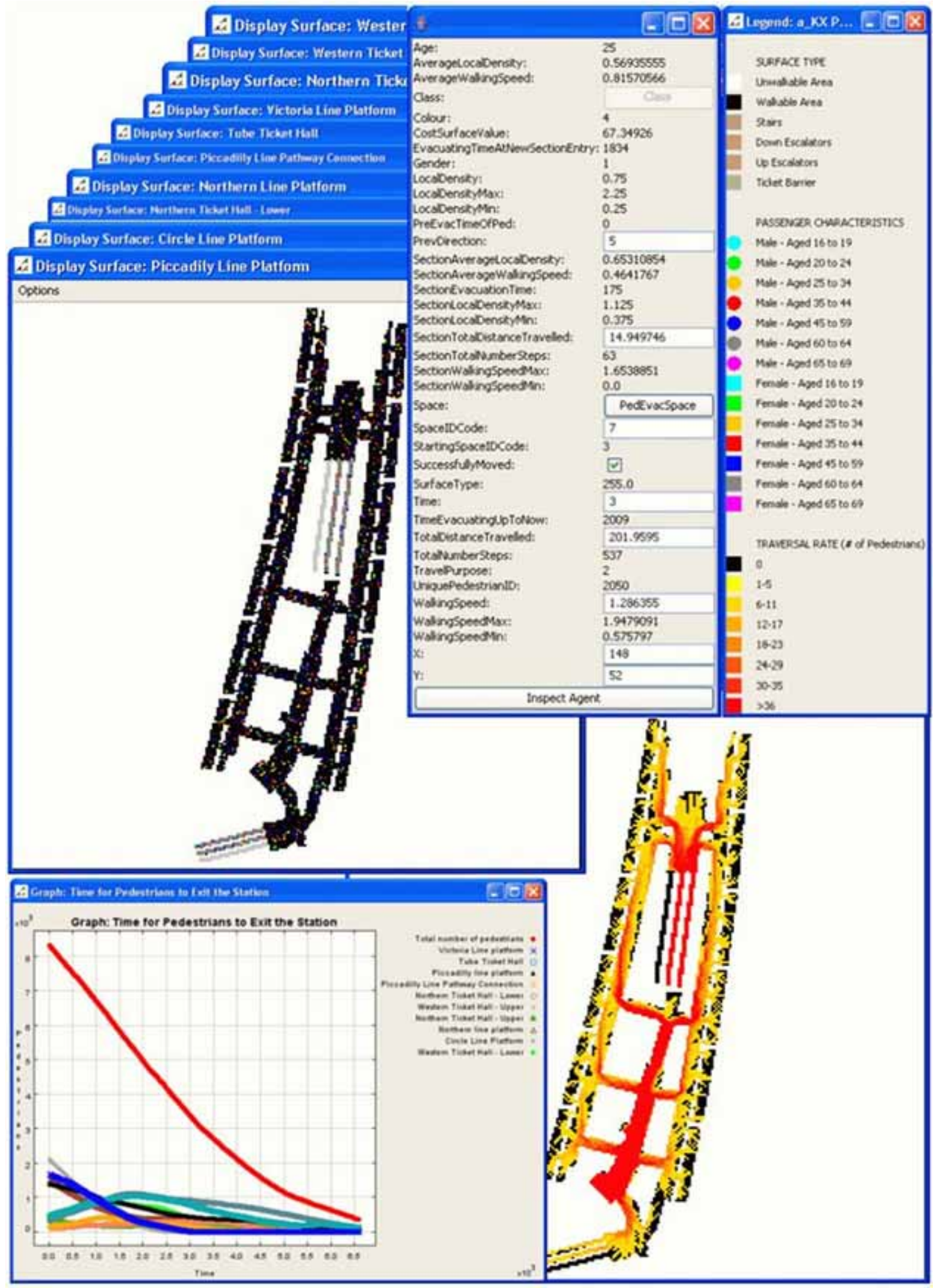

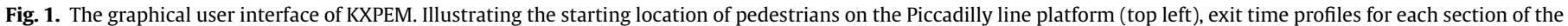
station (bottom left), the accumulative exit path of pedestrians from the Piccadilly line platform (bottom right), and the parameter.

In Fig. 4, we highlight the GUI to the model which like the other applications here provides an essential lens for viewing the opera- tion of the model and judging its results. Clockwise from the top left is the control bar for the simulation, the GIS display which 


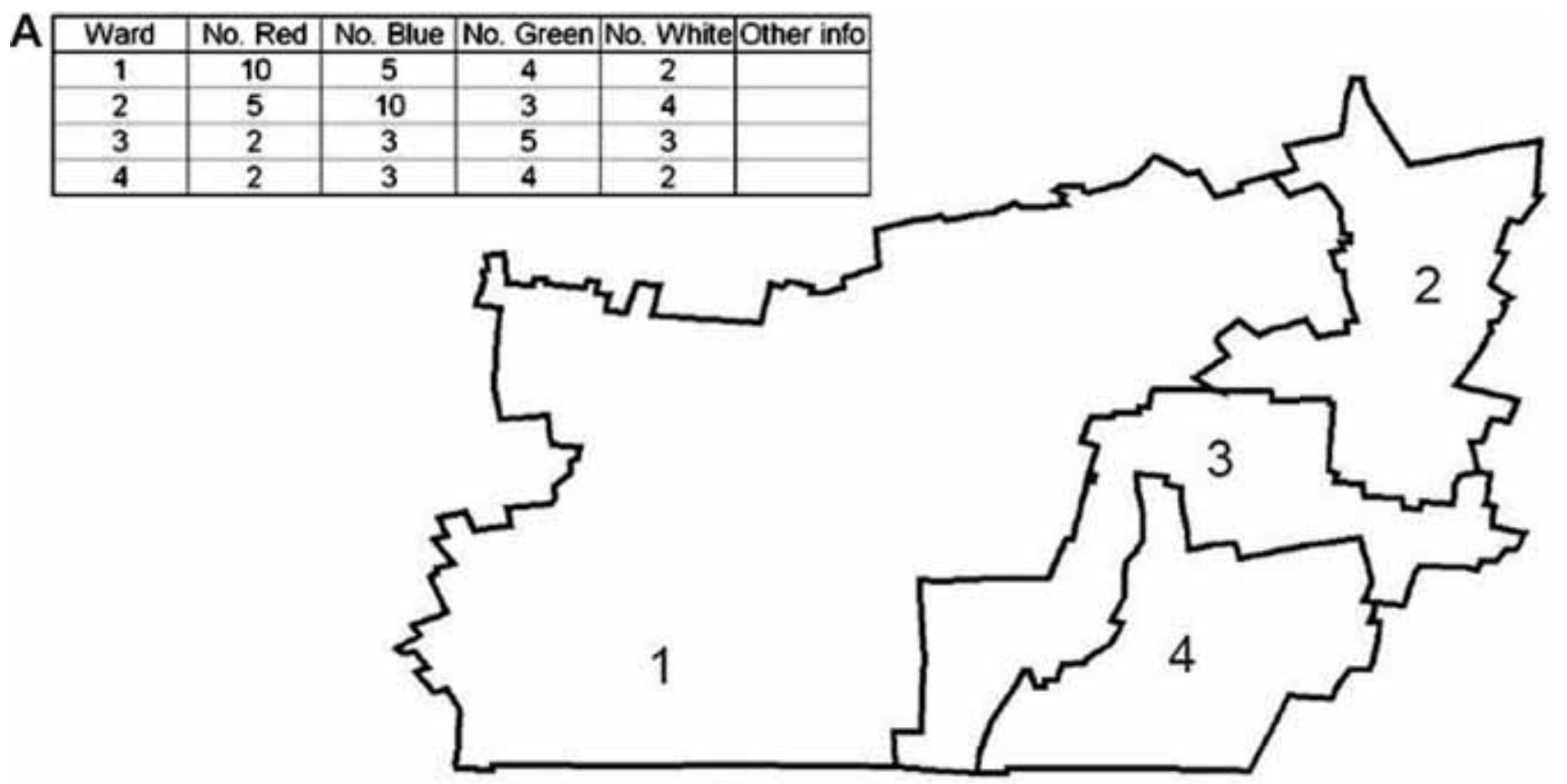

\begin{tabular}{|c|c|c|c|c|c|}
\hline Ward & No. Red & No. Blue & No. Green & No. White & Other info \\
\hline 1 & 10 & 5 & 4 & 2 & \\
\hline 2 & 5 & 10 & 3 & 4 & \\
\hline 3 & 2 & 3 & 5 & 3 & \\
\hline 4 & 2 & 3 & 4 & 2 & \\
\hline
\end{tabular}

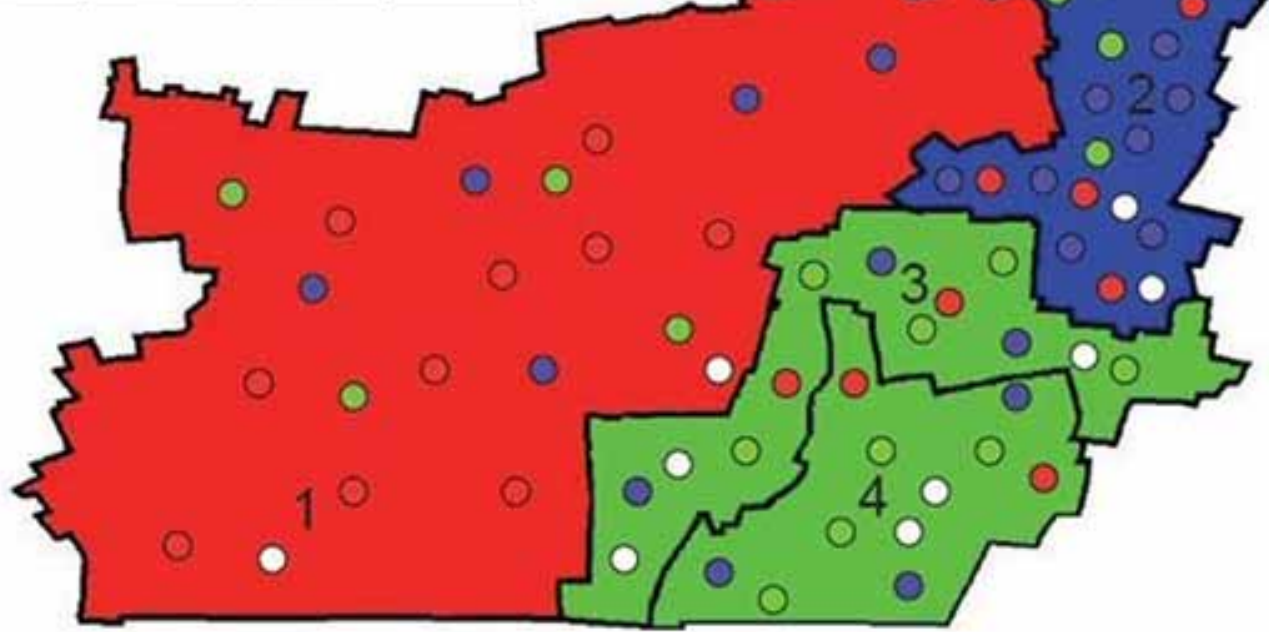

Fig. 2. Reading in the data and creating the agents.

shows the agents and the urban environment (i.e. wards in the City of London), graphs for aggregate outputs, a legend for interpretation of the GIS interface, model output in the form of text, and the model parameters. Although dimensioned to characteristics of populations in Greater London we are not able to validate the model per se, except through testing its plausibility in commonsense terms but we can verify the structure. This is achieved by building the model iteratively similar to KXPEM approach, each step extending the basic model, providing greater realism and functionality. At each step, unit testing was carried out, to ensure no mistake in the computer program had been introduced. This permitted the identification of unexpected outcomes of the model itself as opposed to errors in the code.
Once the model was verified, a series of experiments were carried out in order to test the sensitivity of the model and to highlight the effect of the underlying model assumptions. This exploration provided a detailed understanding of the implications of each assumption but also allowed one to evaluate the logic behind the model. This included the influence of the size of neighbourhoods, the influence of geographical features and the degree to which segregation changes when agent preferences for neighbourhood composition change. These explorations showed that geometry of an area can act as a physical barrier to segregation and that by increasing agents' preferences to reside by a specific group, marked segregation can emerge but not in a linear progression (Crooks, 2007). As with the more 'traditional' segregation 

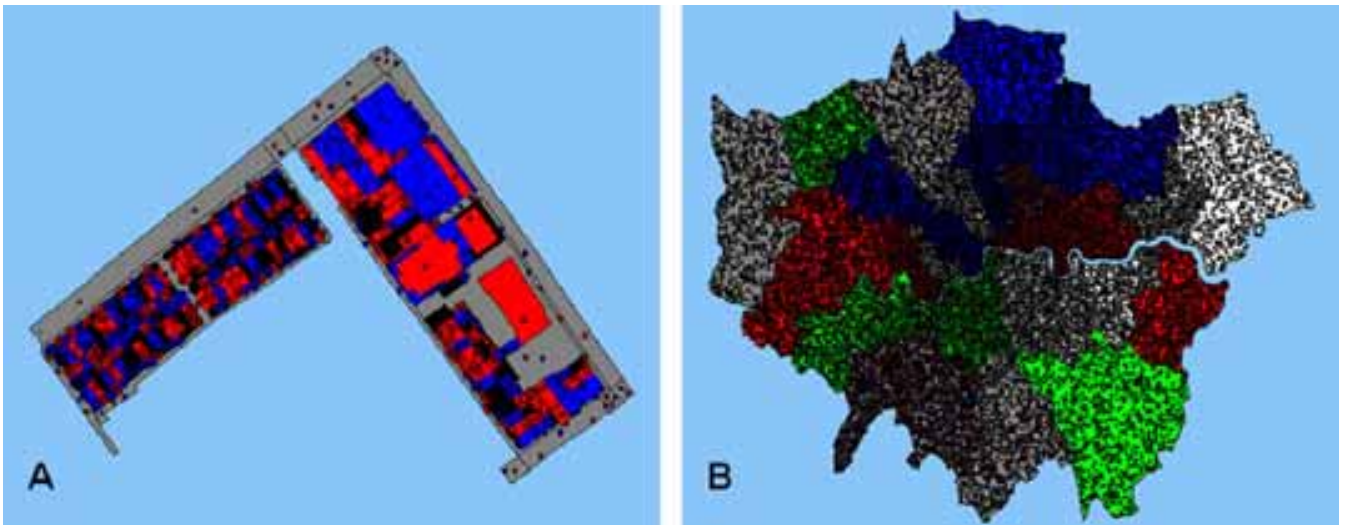

Fig. 3. Spatial representation within the model: (A) a street section, (B) London composed of boroughs. Agents are shown as dots.
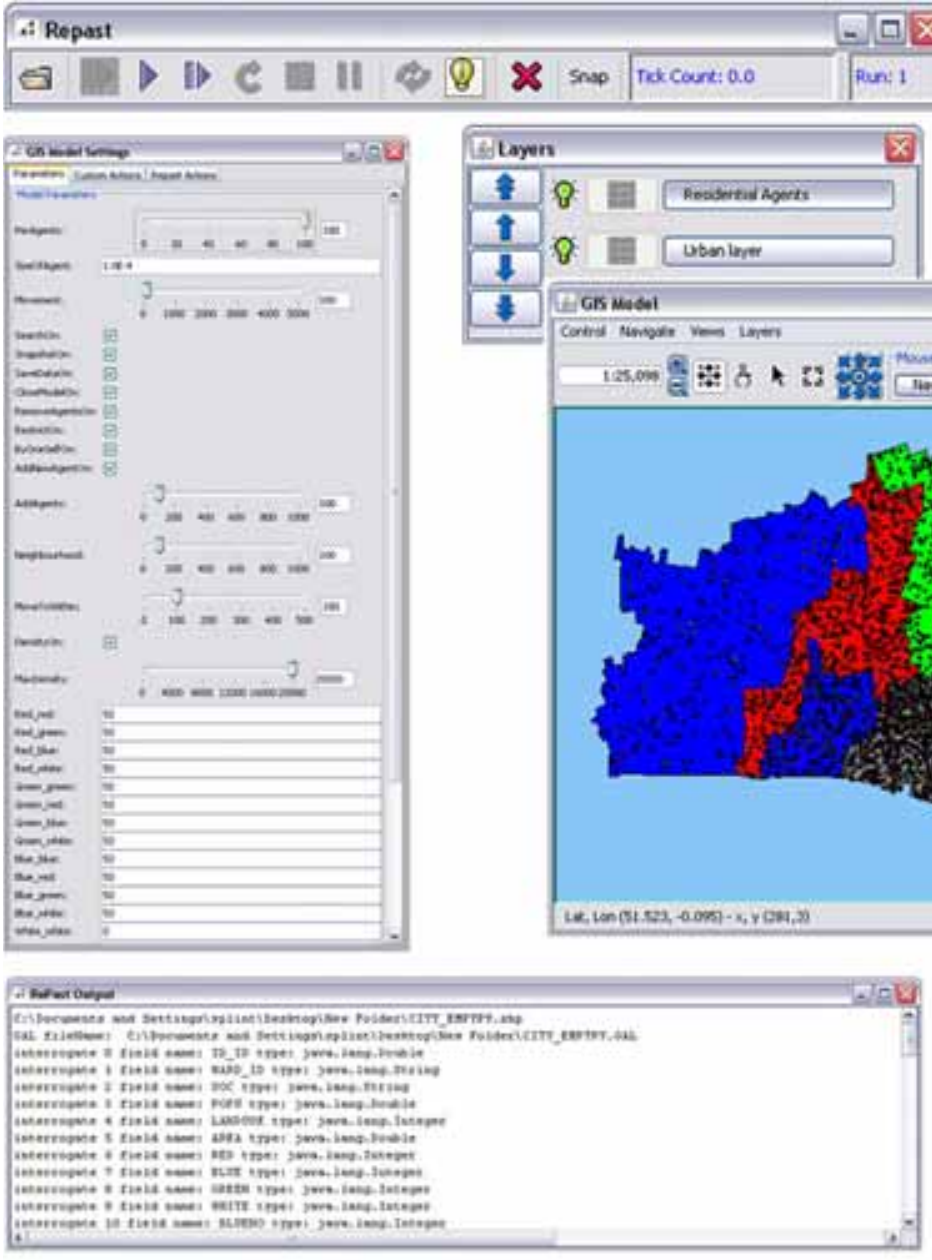
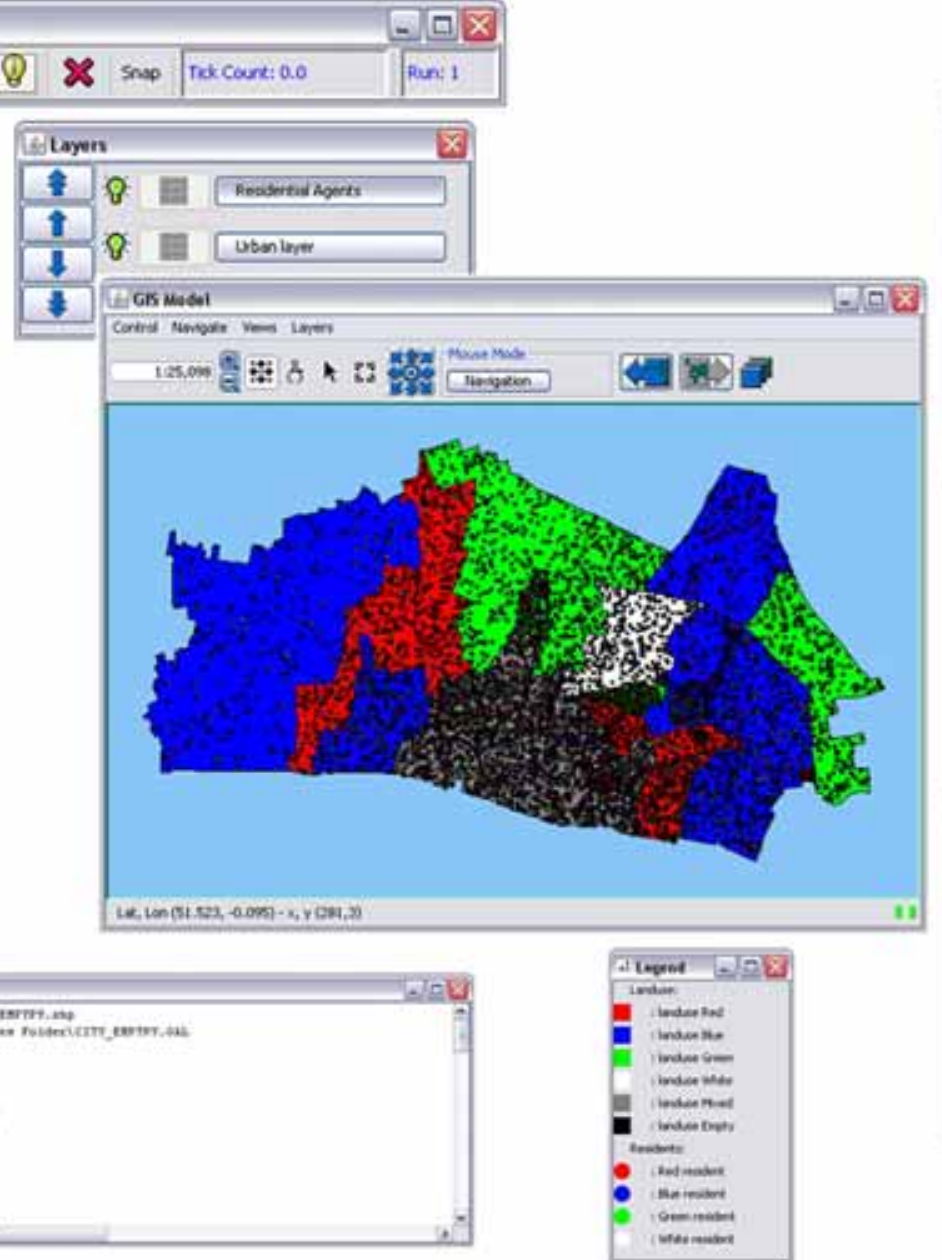
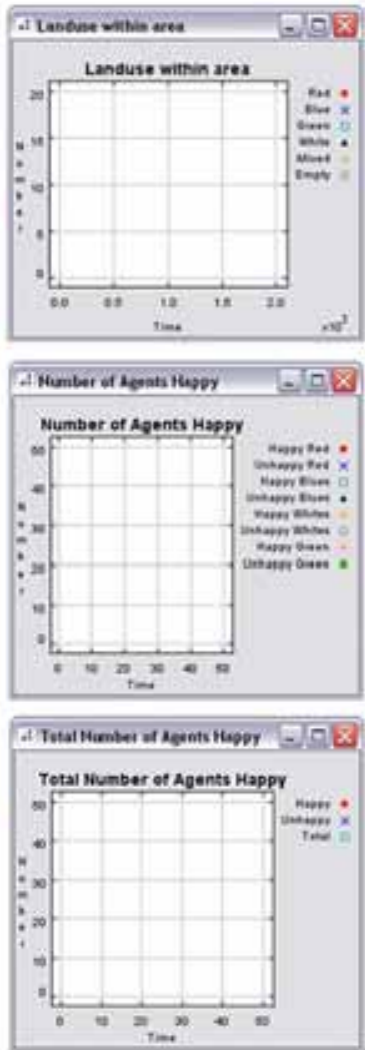

Fig. 4. Segregation model user interface showing segregation dimensioned to the geography of wards in the city of London.

models, this model also highlights how, with mild tastes and preferences to locate amongst 'like' demographic groups, segregation will emerge. Adding agents and removing agents from an existing population alters existing patterns but for new groups entering the system, they must have either low tolerances for other groups or be willing to live by themselves in order to become established and once small minority groups cluster in areas they remain persistent over time. These outcomes are well beyond what Schelling showed in his initial model.
In Fig. 5A, we show a representative simulation outcome where all agents are satisfied with their current neighbourhood locations. While areas may have a predominant type of one agent within them (e.g. a polygon shaded red, has more red agents than any other type), there are areas where there are equal numbers of two or more groups (grey areas). However, closer inspection of these mixed areas in Fig. 5B reveals distinct micro clusters of different types of agents. Moreover it is also clear that clusters do not stop at boundaries but cross them as well and these clusters 


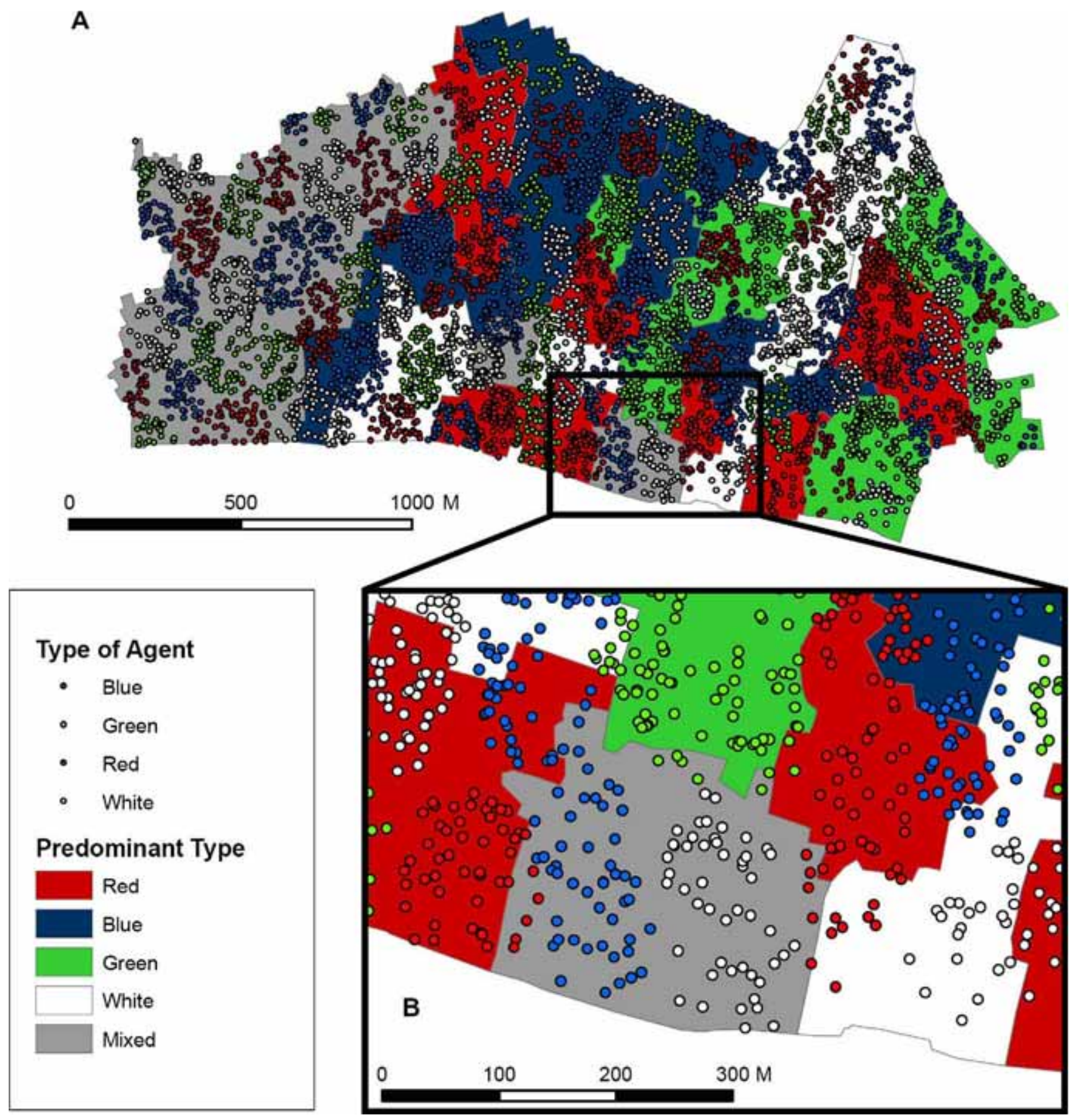

Fig. 5. Segregation within areas and across boundaries: (A) the entire area, and (B) a zoomed in section of A.

would be lost if we were only to consider aggregate level data without the ability of agents to move in free space. This highlights how aggregation can thus confuse our identification of coherent patterns, which we identified as one of the key challenges. Finally this model like KXPEM, is highly visual and modular in its construction. It has been put together using open source software, built around Repast but using GeoTools and OpenMap as well as being coupled to ArcGIS in terms of its inputs and some outputs. It is still only a desktop application but its results are being disseminated and shared across the web which provides a good example of this pedagogy (see http://www.casa.ucl.ac.uk/abm/segregation/) and additionally allows for replication and experimentation through the availability of the source code. It is not designed for policy applications per se although policy is a clear consequence of such thinking. It is a 'classic tool to think with', part of the growing arsenal of techniques and tools useful for informed discussion of urban problems.

\subsection{A residential location model}

Our third application involves a more traditional model of spatial interaction implemented at the level of the individual agents making trips from work to home but articulated at the level of small zones in terms of employment and population aggregates. Such models allocate employment associated with small zones to residential locations, often the same set of small zones, through 
simulating interactions, in this specific case, in the form of the journey to work. The logic of interaction is based on the wellestablished gravitational hypotheses where the flow from employment site to residential location is inversely proportional to some measure of the impedance - distance or travel cost between these origins and destinations, and directly proportional to some measure of attraction or size of each two locations (Batty, 1976). The model is part of an integrated assessment of climate change impacts on the Greater London area. Designed to provide small scale population estimates for long term (2050 and 2100 scenarios) interfacing between higher scale climate predictions and regional employment and population estimates from an environmental input-output model and lower scale models related primarily to flooding and environmental risk (Dawson et al., 2007). The model is also designed to predict trips in four modes of transport - car, bus, rail and tube - which each have a considerable impact on how the pattern of residential population might adjust in terms of the way people might travel in the future.

The purpose of this model is clear and the theory on which it is based is classical social physics. It is also has strong links with urban economic theory and the operation of land markets through the trade-off between accessibility and the attraction of different locations. This sort of model has been replicated many times and because it is comparatively parsimonious, it can be fitted at the aggregate level to available data. Flow matrices for each mode of travel represent the key data for validation and calibration is accomplished through tuning the model to reproduce the known trip lengths for each mode. The key problems with such models relate to the fact that the heterogeneity of location is not represented other than in the distinctions between the small zones used in predicting aggregate trips. Although there are over 600 zones defined for the model (see Fig. 6 where we show the overall data input sequence and work trips from one zone), the fine grained spatial detail which characterises each place is not picked up. To reflect this, we have further disaggregated the model so that the behaviour of each household or trip making entity is represented separately (i.e. the agent component). The 4 million work trips in Greater London, are represented in terms of location but without sacrificing the aggregate conservation properties of the spatial interaction models which enable realistic totals to be predicted.

What we have done is divide each of the 633 zones into a very fine grid with a dimension $1000 \times 800$ (each cell being about $60 \mathrm{~m} \times 60 \mathrm{~m}$ ). We then randomly allocate the known employment and residential totals, individual by individual, to these grid squares but constrained by the actual pattern of development in each zone. The average number of agents in each grid square is a little over one individual but in the densest part of this lattice the number reaches over 3000. Non-residential and employment cells are thereby excluded and this produces a much more accurate pattern of trip making. As the distances used in the model are crow-fly/airline, this detail affects every individual or agent who makes a trip. We are currently developing ways of building in real distance/cost surfaces into the model (using detailed GIS transport networks) so that we can move beyond straight-line distances. Each one of the four million agent trips is then individually simulated in terms of choosing a residential location in an appropriate zone and as each trip is identified in terms of its origin, running the model at the individual agent level conserves the total activity at each origin.

Agents move in that they make trips, and in so far as a dynamics exists, it is simply in the way people respond to fixed locations and spatial impedances which are unvarying. This model is in fact a

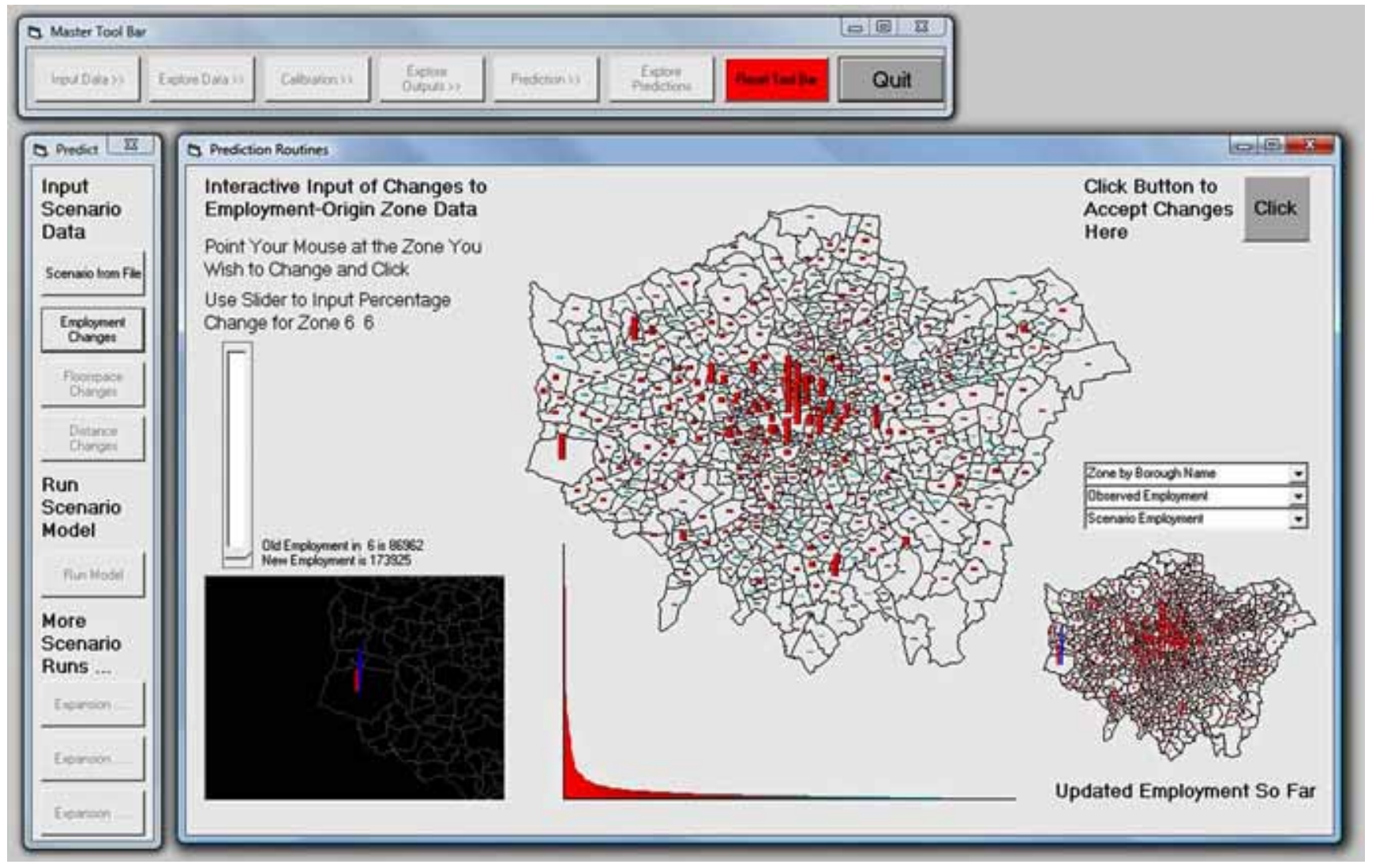

Fig. 6. Inputting and exploring the model's data aggregate work trips from Heathrow to the other 632 destinations are shown along with the zone map and drop-down menus enabling the user to interrogate the data. 


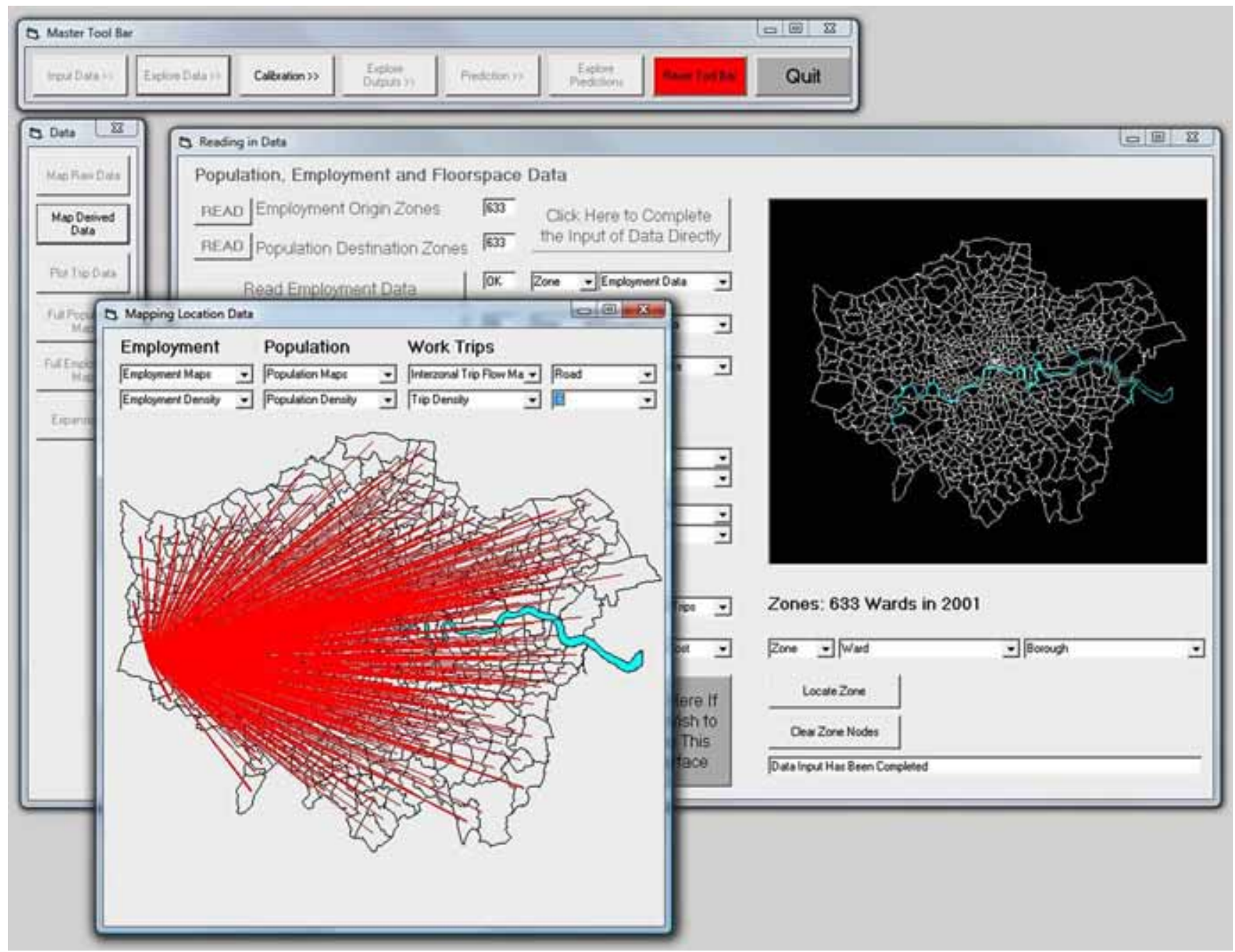

Fig. 7. Calibrating the model. The calibration window, aggregate observed and predicted work trips from Heathrow, and predicted populations are shown.

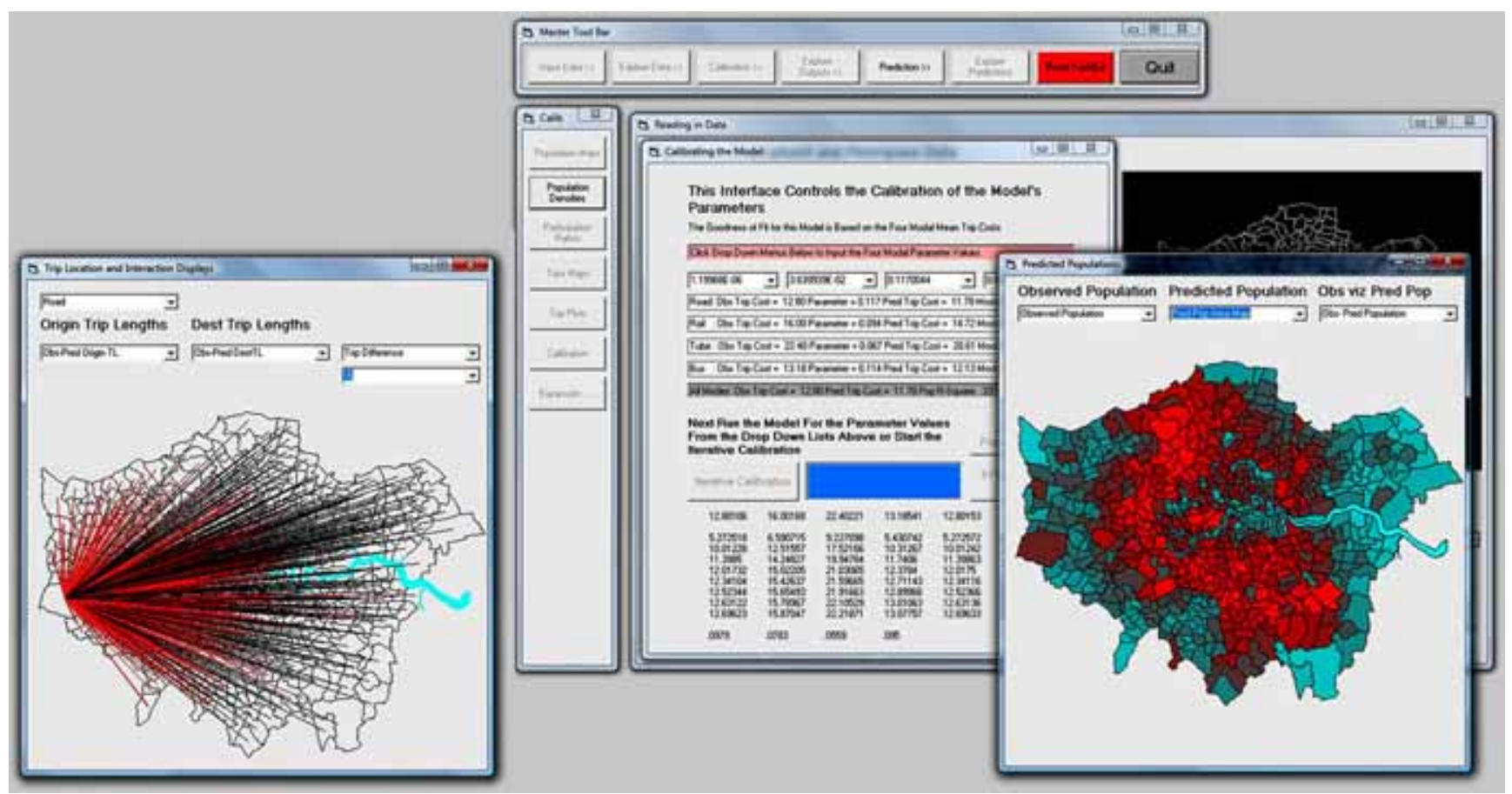

Fig. 8. The interface enabling the user to add or subtract employment from any of the 633 zones in composing a future scenario to test. 
simulation of a static equilibrium, although the equilibrium is composed of individual agents which when aggregated to small zones meet certain conservation constraints. The model is also operational in a somewhat different way from the previous two. The model is not programmed in a particular ABM package but in a standard language -Visual Basic (VB). Its graphics interface and its maps use the components of Visual Studio API. The model is in fact programmed to show how the sequence of model construction, calibration and prediction is ordered and there are many graphics which enable the user to view data and predictions, and even to input various scenarios from the desktop as shown in Fig. 7.

The model can be communicated and shared with others through its graphic interface. Unlike the Repast models, we have not yet sought to embed its output in an online system although we are making movies of how the model can be used for wider dissemination (see http://www.casa.ucl.ac.uk/transportmodel/transportmodel.asp). The main form of communication comes from enabling users to develop their own scenarios. In Fig. 8, we show one part of this interface for inputting new levels of employment in each small zone of the system.

\section{Conclusions}

These models demonstrate how the representation of individuals, through simple rules governing their behaviour and interaction at the micro-scale, can result in recognisable patterns at the macro-scale. The models apply different theories and concepts, highlighting how ideas pertaining to urban phenomena can easily be abstracted within agent-based models, helping further our understanding of how cities operate. Furthermore, these models help laminate the importance of incorporating space when modelling urban systems. Notwithstanding their potential, this class of geo-spatial models more than any developed hitherto raise challenges for the field that directly face the issue about the changing scientific method which is being forced by the development of computation and highly decentralised views of how spatial systems actually work and how such challenges can be addressed. Each of these applications does not address all seven challenges, thus illustrating the limits to any modelling venture in general and $\mathrm{ABM}$ in particular.

One major limitation of agent-based models that we have implicitly noted in our critique is their arbitrariness due to the perceived need to represent the world in as rich a manner as possible. The assumptions behind such models may be quite plausible but with the scale of parameterisation needed to make such models operational, the prospects for their testing in anything but the most cursory way is limited. This means that there will always be a strong degree or arbitrariness to such agent-based models, thus implying that a goal for the field is to switch effortlessly between rich, perhaps untestable models and their simpler equivalents that ensure that the model-builder has some sense of how such models capture the real systems that are being simulated. Once again this style of modelling throws up the tension between parsimony and realism, complexity and simplicity, reinforcing the fine line between good, applicable models and those that have limited value.

The challenges we have identified here are not new for they pertain to all science which seeks to hypothesise the workings of a real system in the quest to develop both better understanding and tools for manipulating it, in silico, so to speak. The major challenge however which emerges from this discussion is the fact that agent-based models can be much more arbitrary than the models they both complement and replace. What is urgently required is some consensus about ways in which ABM can be structured so that major pitfalls are avoided. It is all too easy to develop models whose components seem plausible but are in fact rooted in false intuitions and unwarranted assumptions. Much of this relates to the goal of science which traditionally has been simplification but is changing to embrace possibilities for theories that are too rich to test but essential for coping with the evident complexity of the systems under scrutiny. Cities tend to be key exemplars of the dilemmas faced in such modelling, and the clear but short conclusion of this paper is that all such models should come under a much greater degree of scrutiny than any hitherto to avoid the sins of arbitrariness that plague a world where there are almost as many models as modellers. This represents a continuing challenge.

\section{Acknowledgements}

We gratefully acknowledge the economic support from the Economic and Social Research Council, the Greater London Authority Economics Unit, and NERC, through the Tyndall Centre for Climate Change.

\section{References}

Ando, K., Ota, H., \& Oki, T. (1988). Forecasting the flow of people. Railway Research Review, 45, 8-14

Axelrod, R. (2007). Simulation in the social sciences. In J.-P. Reynard (Ed.), Handbook of research on nature inspired computing for economy and management (pp. 90-100). Hershey, CA: Idea Group.

Axtell, R., Axelrod, R., Epstein, J. M., \& Cohen, M. D. (1996). Aligning simulation models: A case study and results. Computational and Mathematical Organization Theory, 1(1), 123-141.

Batty, M. (1976). Urban modelling: Algorithms, calibrations, predictions. Cambridge, UK: Cambridge University Press.

Batty, M. (2005). Cities and complexity: Understanding cities with cellular automata, agent-based models, and fractals. Cambridge, MA: The MIT Press.

Batty, M. (2008). Fifty years of urban modelling: Macro statics to micro dynamics. In S. Albeverio, D. Andrey, P. Giordano, \& A. Vancheri (Eds.), The dynamics of complex urban systems: An interdisciplinary approach (pp. 1-20). Heidelberg, DE: Physica-Verlag.

Batty, M., \& Torrens, P. M. (2005). Modelling and prediction in a complex world. Futures, 37(7), 745-766.

Batty, M., Steadman, P., \& Xie, Y. (2006). Visualization in spatial modelling. In J. Portugali (Ed.), Complex artificial environments (pp. 49-70). Berlin: Springer.

Benenson, I., \& Torrens, P. M. (2004). Geosimulation: Automata-based modelling of urban phenomena. London: John Wiley \& Sons.

Benenson, I., Omer, I., \& Hatna, E. (2002). Entity-based modelling of urban residential dynamics: The case of Yaffo, Tel Aviv. Environment and Planning $B$ Planning and Design, 29(4), 491-512.

Birkin, M., Turner, A., \& Wu, B. (2006). A synthetic demographic model of the UK population: Methods, progress and problems. In Regional Science Association International British and Irish Section, 36th Annual Conference, The Royal Hotel, St Helier, Jersey, Channel Islands.

Brown, D. G. (2006). Agent-based models. In H. Gist (Ed.), The earths changing land: An encyclopaedia of land-use and land-cover change (pp. 7-13). Westport, CN: Greenwood Publishing Group.

Brown, D. G., Riolo, R., Robinson, D. T., North, M., \& Rand, W. (2005). Spatial process and data models: Toward integration of agent-based models and GIS. Journal of Geographical Systems, 7(1), 25-47.

Bruch, E. E., \& Mare, R. D. (2005). Neighbourhood choice and neighbourhood change. Los Angeles, CA: California Centre for Population Research University of California - Los Angeles.

Casti, J. L. (1997). Would-be-worlds: How simulation is changing the frontiers of science. New York: John Wiley \& Sons.

Castle, C. J. E. (2007a). Agent-based modelling of pedestrian evacuation: A study of London's King's Cross underground station. PhD Thesis, University College London.

Castle, C. J. E. (2007b). Guidelines to assess pedestrian evacuation software applications. Working paper 115. London: Centre for Advanced Spatial Analysis (University College London).

Castle, C. J. E., \& Longley, P. A. (2008). Emergency pedestrian evacuation analysis: A review and interpretation of software applications. In D. Z. Sui \& S. L. Cutte (Eds.), Geospatial technologies and homeland security: Research frontiers and challenges (pp. 209-228). Berlin: Springer.

Castle, C. J. E., \& Crooks, A. T. (2006). Principles and concepts of agent-based modelling for developing geospatial simulations. Working paper 110. London: Centre for Advanced Spatial Analysis (University College London).

Couclelis, H. (2002). Modelling frameworks, paradigms, and approaches. In K. C. Clarke, B. E. Parks, \& M. P. Crane (Eds.), Geographic information systems and environmental modelling. London: Prentice Hall. 
Crooks, A. T. (2007). Experimenting with cities: Utilizing agent-based models and GIS to explore urban dynamics. PhD Thesis, University College London.

Dawson, R. J., Hall, J. W., Barr, S., Batty, M., Bristow, A., \& Carney, S., et al. (2007). A blueprint for the integrated assessment of climate change in cities. Tyndall Centre Working paper 104. UK: University of East Anglia, Norwich. Available at http://www.tyndall.ac.uk/publications/working_papers/twp104.pdf.

Epstein, J. M., \& Axtell, R. (1996). Growing artificial societies: Social science from the bottom up. Cambridge, MA: MIT Press.

Fruin, J. J. (1971). Pedestrian planning and design. New York: Metropolitan Association of Urban Designers and Environmental Planners.

Galea, E. R., \& Gwynne, S. (2006). Principles and practice of evacuation modelling (7th ed.). London: Fire Safety Engineering Group - University of Greenwich.

Gilbert, N., den Besten, M., Bontovics, A., Craenen, B. G. W., Divina, F., Eiben, A. E., et al. (2006). Emerging artificial societies through learning. Journal of Artificial Societies and Social Simulation, 9(2), 9.

Grimm, V., Berger, U., Bastiansen, F., Eliassen, S., Ginot, V., Giske, J., et al. (2006). A standard protocol for describing individual-based and agent-based models. Ecological Modelling, 198, 115-126.

Hankin, B. D., \& Wright, R. A. (1958). Passenger flow in subways. Operational Research Quarterly, 9(2), 81-88.

HSE (1996). Railway safety principles and guidance: Part 2 - Section B - Guidance on stations. London: Health \& Safety Executive.

Langran, G. (1992). Time in geographic information systems. London: Taylor and Francis.

Law, A. M., \& Kelton, D. (1991). Simulation modelling and analysis (2nd ed.). New York: McGraw-Hill.

Lee, D. B. (1973). Requiem for large-scale models. Journal of the American Institute of Planners(39), 163-178.

Longley, P. A., Goodchild, M. F., Maguire, D. J., \& Rind, D. W. (2005). Geographical information systems and science. New York: John Wiley \& Sons.

Maguire, D. J. (2005). Towards a GIS platform for spatial analysis and modelling. In D. J. Maguire, M. Batty, \& M. F. Goodchild (Eds.), GIS, spatial analysis and modelling (pp. 19-39). California, CA: ESRI Press, Redlands.

Mandelbrot, B. B. (1983). The fractal geometry of nature. New York: W.H. Freeman.

North, M. J., \& Macal, C. M. (2007). Managing business complexity: Discovering strategic solutions with agent-based modeling and simulation. New York: Oxford University Press.
O’Sullivan, D., MacGill, J., \& Yu, C. (2003) Agent-based residential segregation: A hierarchically structured spatial model. In Proceedings of agent 2003 conference on challenges in social simulation, The University of Chicago, Chicago, IL, pp. 493507.

Parker, D. C. (2005). Integration of geographic information systems and agent-based models of land use: Challenges and prospects. In D. J. Maguire, M. Batty, \& M. F. Goodchild (Eds.), GIS, spatial analysis and modelling (pp. 403-422). Redlands, CA: ESRI Press.

Peuquet, D. J. (2005). Time in GIS and geographical databases. In P. A. Longley, M. F. Goodchild, D. J. Maguire, \& D. W. Rhind (Eds.), Geographical information systems: Principles, techniques, management and applications (Abridged Edition). New York: John Wiley \& Sons.

Polhill, J. G., Parker, D., Brown, D., \& Grimm, V. (2008). Using the ODD protocol for describing three agent-based social simulation models of land-use change. Journal of Artificial Societies and Social Simulation, 11(2), 3 http:// jasss.soc.surrey.ac.uk/11/2/3.html.

Pontius, R. G., \& Malanson, J. (2005). Comparison of the structure and accuracy of two land change models. International Journal of Geographical Information Science, 19, 243-265.

Predtechenskii, V. M., \& Milinskii, A. I. (1978). Planning for foot traffic flow in buildings. New Delhi: Amerind.

Railsback, S. F., Lytinen, S. L., \& Jackson, S. K. (2006). Agent-based simulation platforms: review and development recommendations. Simulation, 82(9), 609-623.

Schelling, T. C. (1971). Dynamic models of segregation. Journal of Mathematical Sociology, 1, 143-186.

Tobias, R., \& Hofmann, C. (2004). Evaluation of free Java-libraries for social-scientific agent based simulation. Journal of Artificial Societies and Social Simulation, 7(1), 10 .

Webster, F. V., Bly, P. H., \& Paulley, N. J. (Eds.). (1988). Urban land-use and transport interaction: Policies and models. Report of the International Study Group on Land-Use/Transport Interaction (ISGLUTI), Avebury, Aldershot, UK.

Westervelt, J. D. (2002). Geographic information systems and agent-based modelling. In H. R. Gimblett (Ed.), Integrating geographic information systems and agent-based modelling techniques for simulating social and ecological processes (pp. 83-104). Oxford, UK: Oxford University Press. 